\title{
STUDY OF THE PREVALENCE OF VANCOMYCIN RESISTANT AS WELL AS HIGH LEVEL GENTAMICIN RESISTANT ENTEROCOCCI IN VARIOUS CLINICAL SPECIMENS AT MGM MEDICAL COLLEGE AND LSK HOSPITAL, KISHANGANJ, BIHAR
}

\author{
Nibedita Haldar1, Santwana Chattopadhyay², Atanu Roy3 \\ 1 Professor, Department of Microbiology, MGM Medical College \& LSK Hospital, Kishanganj, Bihar. \\ 2 Postgraduate Student, Department of Microbiology, MGM Medical College \& LSK Hospital, Kishanganj, Bihar. \\ ${ }^{3}$ Professor and HOD, Department of Microbiology, MGM Medical College \& LSK Hospital, Kishanganj, Bihar.
}

\begin{abstract}
\section{BACKGROUND}

For many years Enterococcus species were believed to be harmless commensals of human beings. During the past decade, there has been a worldwide trend in increasing occurrence of Enterococci in the hospitals, a shift in the spectrum of Enterococcal infections and emergence of antimicrobial resistance among such isolates. The steady Pandemic spread of Vancomycin Resistant Enterococci (VRE) along with acquisition of High Level Gentamicin Resistance (HLGR), $\beta$-lactamase production and glycopeptides resistance warrants continued surveillance of these versatile pathogens.
\end{abstract}

\section{MATERIALS AND METHODS}

Therefore, the present study has been carried out on a total no of 2788 clinical specimens (Urine, Pus, Blood and Cerebrospinal fluid) received from different indoor and outpatient departments (Medicine, Surgery, Orthopaedics, Gynaecology \& Obstetrics, Paediatrics, Tuberculosis and Chest departments) of Mata Gujri Memorial Medical College and Lions Seva Kendra Hospital, Kishanganj, Bihar over a period from 1st Oct 2015 up to 30th Sept 2016. Various samples were processed using standardized technique and cultures were done on Blood agar, MacConkey agar, Hi-chrome agar, Brain Heart Infusion (BHI) broth for blood culture. From the colonies on MacConkey media and Hi-chrome agar after performing Gram staining and motility testing were done by hanging drop preparation after subculturing the growth in nutrient broth and overnight incubation at $37^{\circ} \mathrm{C}$. Various biochemical tests were done with the suspected colony for final identification of Enterococci and its identification at species level. Antibiotic Susceptibility testing was done by Kirby Bauer disc diffusion method as per CLSI guideline using $30 \mu \mathrm{g}$ vancomycin disc and $120 \mu \mathrm{g}$ Gentamicin Disc. Minimum inhibitory concentration (MIC) was done by Macrodilution method and E test.

\section{RESULTS}

Out of a total of 2788 clinical specimens, growth of organisms was found in $2400(86.08 \%)$ specimens. Prevalence of Enterococci among the total no of growth positive specimens were 280 (11.67\%). Number of VRE among 280 Enterococci found in various clinical specimens were 24 (8.57\%). Species of VRE were Enterococcus (E) faecalis 15 (62.50\%), E faecium 9 (37.50\%). Total no of HLGR Enterococci among 280 Enterococcal growth was 100 (35.71\%) which includes 20 VRE strains.

\section{CONCLUSION}

CDC's new guidelines for prevention and control of these infections should be strictly followed.

\section{KEYWORDS}

Vancomycin Resistant Enterococci, High Level Gentamicin Resistant, Enterococcus, Mueller Hinton Agar, Brain Heart Infusion, Minimum Inhibitory Concentration, Out-Patient Department, In Patient Department.

HOW TO CITE THIS ARTICLE: Haldar N, Chattopadhyay S, Roy A. Study of the prevalence of vancomycin resistant as well as high level gentamicin resistant enterococci in various clinical specimens at MGM medical college and LSK Hospital, Kishanganj, Bihar. J. Evolution Med. Dent. Sci. 2017;6(11):850-856, D0I: 10.14260/Jemds/2017/184

\section{BACKGROUND}

The war between drugs and bugs has been on since the time of Paul Ehrlich, father of Chemotherapy followed by the subsequent discovery of Penicillins in 1929 by Alexander Fleming. It is now known that the frequent flow of genetic materials across the whole bacteria species is an inevitable phenomenon that keep happening in nature as part of natural

Financial or Other, Competing Interest: None.

Submission 27-12-2016, Peer Review 21-01-2017,

Acceptance 27-01-2017, Published 06-02-2017.

Corresponding Author:

Dr. Nibedita Haldar,

Ramkrishna Road $1^{\text {st }}$ by Lane,

J. K. Dutta Sarani, Ashrampara,

Siliguri-734001, Darjeeling, West Bengal.

E-mail: nibeditaslg@yahoo.com

DOI: $10.14260 /$ jemds $/ 2017 / 184$ selection. It could just happen anywhere \& anytime. Enterococci were originally classified as enteric grampositive cocci and later included in the genus Streptococcus. In 1930s, with the establishment of the Lancefield serological typing system, enterococci were classified as group D streptococci and were differentiated from the nonenterococcal group D Streptococci such as Streptococcus bovis by distinctive biochemical characteristics. Sherman further recommended that the term "enterococcus" should be used specifically for streptococci that grow at both 10 and $45^{\circ} \mathrm{C}$, at $\mathrm{pH} 9.6$, in $6.5 \% \mathrm{NaCl}$ and survive at $60^{\circ} \mathrm{C}$ for 30 mins. These organisms were also noted to hydrolyze esculin in the presence of bile. In the I980s, based on genetic differences, Enterococci were removed from the genus Streptococcus and placed in their own genus, 'Enterococcus'. The previously used species designation such as faecalis, faecium, durans, and so forth were retained but were preceded by the genus 
name Enterococcus in place of Streptococcus. More than 28 Enterococcal species(1) have been identified, only two are responsible for the majority of human infections. Until recently, E faecalis had been the predominant Enterococcal species, accounting for 80 to $90 \%$ of all clinical isolates, and $\mathrm{E}$. faecium had accounted for 5 to $10 \%$. Other Enterococcal species (E. gallinarum, E. casseliflavus, E. durans, E. avium, and E. raffinosus) are isolated much less frequently.(2)

For many years Enterococcus species were believed to be harmless to humans and considered unimportant medically, Recently, Enterococci have become one of the most common nosocomial pathogens, with patients having a high mortality rate of up to $61 \%$ (Dc Fatima Silva Lopes et al 2005). Enterococci have been recognised as an important cause of endocarditis for almost a century. In addition to this longestablished role, Enterococci began to be recognized as common causes of hospital-acquired infections in the middle to late 1970s. This was coincident with and probably related to the increasing use of third-generation Cephalosporins to which Enterococci are naturally resistant. Recently VRE and multi drug resistant Enterococci including HLGR Enterococci have been emerging as a cause of nosocomial super infection in surgical and intensive care unit particularly organ transplant. Beside nosocomial infection of surgical wounds, VRE and multi drug resistant enterococci are emerging causes of meningitis in neonates as well as infections of central nervous system, osteomyelitis, lung infection, urinary tract and pelvic infection in adults. (3)

In 2005 there were 7066 reported cases of bacteraemia caused by Enterococcus species in the UK. Twenty-eight percent of all cases were antibiotic resistant (Health Protection Agency, 2007). The risk of death from VRE infection is $75 \%$, compared with $45 \%$ for those infected with a susceptible strain (Bearman \&Wenzel, 2005). These figures are mirrored in the USA. Over a 15 year period there was a 20 -fold increase in VRE associated with nosocomial infections reported to CDC's National Nosocomial Infections Surveillance, 2004)(4) In 2003, Purba Mathur et al(5) from AIIMS, New Delhi, India, reported 444 isolates of E. faecalis from various clinical samples among which $26 \%$ were HLGR and 1\% VRE. In 2012 Anjana Telkar et al(6) from J.J.M. Medical College, Karnataka, India reported 50 Enterococci isolates from blood culture. In their report vancomycin resistance was seen in $6(12 \%)$ of the enterococci isolates and $34(68 \%)$ isolates were HLGR. The dramatic increase in antibiotic resistant Enterococcus Species worldwide highlights the need for a greater understanding of this genus, including its ecology and epidemiology. Epidemiology of VRE and HLGR Enterococcal infections have been studied in several hospitals in different corners of globe including parts of India(7) However, there is a scarcity of information on actual prevalence data on VRE \& HLGR Enterococci in clinical specimens of eastern parts of India, like Bihar and West Bengal. Therefore, the present study has been undertaken to assess the actual prevalence of vancomycin resistance and high level gentamicin resistance in Enterococci isolated from various specimens of patients at M.G.M. Medical College and LSK Hospital, Kishanganj, Bihar.

\section{MATERIALS AND METHODS}

Present study was carried out on a total of 2788 clinical specimens (Urine, Pus, Blood \& Cerebrospinal fluid) received from different indoor and outpatient departments (OPDs) (Medicine, Surgery, Orthopaedics, Gynaecology \& Obstetrics, Paediatrics, Tuberculosis \& Chest) of Mata Gujri Medical College and LSK Hospital, Kishanganj, Bihar over a period from 1st Oct. 2015-30th Sept. 2016. Various samples were processed using standardised technique and culture were done on Blood agar, MacConkey agar, HiCrome agar, BHI broth for blood culture. From the colony on MacConkey media \& Hi-Chrome agar after performing Gram staining Motility testing were done by hanging drop preparation after subculturing the growth on the nutrient broth \& overnight incubation at $37^{\circ} \mathrm{C}$. Various biochemical tests were done with the suspected colony for final identification of Enterococci eg., catalase test, Pyrase (L-pyrrolidonyl arylamidase) test, Bileesculin test, Salt-tolerance test with $6.5 \% \mathrm{NaCl}$ broth, growth at $10^{\circ} \mathrm{C}$ and $45^{\circ} \mathrm{C}$, growth at $9.6 \mathrm{pH}$, Heat tolerance test at $60^{\circ} \mathrm{C}$ for 30 minutes in a water bath. Various species identification of Enterococci were done using various biochemical tests like mannitol, Sorbitol, arginine, arabinose, raffinose, $0.04 \%$ tellurite, sucrose, pyruvate, pigment production.(8) Identification of Enterococcus species has also been confirmed by Vitek 2 (Automated system).

\section{Antibiotic Susceptibility Test}

In this study sensitivity to vancomycin and high Level Gentamicin were performed for isolated Enterococci. Antibiotic susceptibility testing was done in Mueller Hinton agar (MHA) plate by Kirby Bauer disc diffusion method as per CLSI guidelines. Susceptibility to Vancomycin was performed by $30 \mu \mathrm{g}$ disc and Susceptibility to High Level Gentamicin was done by $120 \mu$ g disc.

The zone of inhibition size was interpreted according to CLSI guidelines. Zone was examined using transmitted light for vancomycin.

\begin{tabular}{|c|c|c|c|}
\hline \multirow{2}{*}{} & \multicolumn{3}{|c|}{$\begin{array}{c}\text { Zone Diameter Interpretive } \\
\text { Criteria nearest whole mm }\end{array}$} \\
\hline Disc content & $\mathrm{S}$ & $\mathrm{I}$ & $\mathrm{R}$ \\
\hline $30 \mu \mathrm{g}$ & $\geq 17$ & $15-16$ & $\leq 14$ \\
\hline \multicolumn{3}{|c|}{ Zone Diameter for Vancomycin } \\
\hline
\end{tabular}

$\mathrm{S}=$ Sensitive I= Intermediate $\mathrm{R}=$ resistant.

\begin{tabular}{|c|c|c|c|}
\hline \multirow{2}{*}{ Disc Content } & \multicolumn{3}{|c|}{$\begin{array}{c}\text { Zone Diameter Interpretive } \\
\text { Criteria nearest whole mm }\end{array}$} \\
\hline \multirow{2}{*}{$120 \mu \mathrm{g}$} & $\mathrm{S}$ & $\mathrm{I}$ & $\mathrm{R}$ \\
\cline { 2 - 4 } & $\geq 10$ & $7-9$ & 6 \\
\hline \multicolumn{2}{|c|}{ Zone Diameter for High Level Gentamicin (HLG) } \\
\hline
\end{tabular}

$\mathrm{S}=$ Sensitive I= Intermediate $\mathrm{R}=$ resistant.

Vancomycin resistant and intermediate resistant Enterococci found by disc diffusion method were screened by vancomycin agar screening method.

\section{Vancomycin Agar Screening Method}

MHA plates were prepared with vancomycin $6 \mu \mathrm{g} / \mathrm{ml}$. Suspension of overnight growth of Enterococci in peptone water matched with $0.5 \mathrm{McFarland}$ suspension standard were streaked with standard loop on agar surface and incubated at $37^{\circ} \mathrm{C}$ for $24 \mathrm{hrs}$. 


\section{RESULT}

Growth of 1 colony or more is presumptive of VRE.

VRE alert Kit (Hi Media) also used for screening test.

\section{VRE Alert KIT (Hi Media)}

Tube containing dehydrated media was rehydrated with sterile suspension fluid provided with the kit up to mark (4 $\mathrm{ml})$. Swab soaked with enterococci containing nutrient broth was inoculated in this tube and incubated at $35^{\circ} \mathrm{C}$ for 4 to 8 hrs. Black colouration means esculin hydrolysis positive. Now cap of the tube was replaced with PYR disc containing cap provided with the kit and shook. Positive esculin hydrolysis and red colour change of cap indicated VRE.

\section{MIC Done for Presumptive VRE}

MIC: It is the minimum concentration of anti-biotic which inhibit the growth of bacteria in a liquid media on visual interpretation.

In this study MIC done by (i) Macro dilution (tube dilution) (ii) E-test.

\section{Macro Dilution Method(9)}

Each tube containing antibiotic in two-fold dilution one after another was inoculated with inoculum (0.5 McFarland standard) and incubated at $37^{\circ} \mathrm{C}$ for $24 \mathrm{hrs}$ and looked for turbidity or growth of organism. The tube which showed clear i.e. no turbidity with minimum antibiotic concentration was called MIC of that antibiotic for that organism. Media used for macro dilution was BHI broth.

Vancomycin concentration were $512 \mu \mathrm{g} / \mathrm{ml}, 256 \mu \mathrm{g} / \mathrm{ml}$, $128 \mu \mathrm{g} / \mathrm{ml}, 64 \mu \mathrm{g} / \mathrm{ml}, 32 \mu \mathrm{g} / \mathrm{ml}, 16 \mu \mathrm{g} / \mathrm{ml}, 8 \mu \mathrm{g} / \mathrm{ml}, 4 \mu \mathrm{g} / \mathrm{ml}$ in the tubes no. 8 to 1 respectively and control tube contained no antibiotic and $10^{\text {th }}$ tube contained $2 \mu \mathrm{g} / \mathrm{ml}$ vancomycin in BHI broth but no organism.

Lowest concentration of antibiotics showing no turbidity visually was MIC for the study organism.

\section{E-Test}

The E-Test (Biomerieux, Durhan, North Carolina) used plastic strips, one side of the strip containing the antimicrobial agent concentration gradient and the other containing a numeric scale that indicated the drug concentration. Mueller Hinton plate were inoculated as per disc diffusion method and the strips were placed on the inoculated plates. Several strips could be placed radially on the same plates so that multiple antimicrobials could be tested against a single isolate.

After overnight incubation at $35-37^{\circ} \mathrm{C}$ the plate was examined and the number present at the point where the border of growth inhibition intersected the E-strip was taken as the MIC. In this study vancomycin Himedia-Hicomb E-test strip was used.

CLSI guidelines were followed with E-test value to assign an interpretive category of sensitive, intermediate or resistant.

\section{RESULTS}

Total no. of clinical specimens received at laboratory during period of study: 2788. All 2788 specimens were put on culture for growth of organisms. No growth of organisms was found in 388 (13.92\%) specimens. Growth of organisms was found in $2400(86.08 \%)$ specimens.
Prevalence of Enterococci \& other organisms in total no. of growth positive specimens were as follows: E. coli-30.33\% were the peak, followed by Staphylococci-20.50\%, Klebsiella-16.50\%, Enterococci-11.67\%, Pseudomonas$10.50 \%$, Proteus $-5 \%$, Acinetobacter-2\%, Candida-2\% and Corynebacterium species-0.5\%. (Table 1 )

Among the growth positive samples, Urine samples were 70.83\%, Pus-14.58\%, Blood-10.42\% \& CSF-4.17\%. (Table 2)

Among the species of Enterococcus E. faecalis was top in the list (71.43\%), followed by E. faecium (25.00\%), E. durans \& E. casseliflavus (1.43\% each) and E. gallinarum $(0.71 \%)$. (Table 3).

Distribution of Enterococci found in Out-Patient Department (OPD) and In Patient Department (IPD).

Among 280 Enterococci, 14(5\%) found in samples received from OPD patients and 266 (95\%) found in samples received from IPD. No. of VRE among 280 Enterococci found in various clinical specimens were $24(8.57 \%)$. Species of VRE: E. faecalis: 15 (62.50\%), E. faecium: 9 (37.50\%). Among 280 enterococci E. faecium were 70 (25\%), but among 24 VRE 9 (37.50) were E. faecium. Therefore, prevalence of Vancomycin Resistance is increasing in E. faecium.

Among 24 VRE, 10(41.67\%) found in Urine samples (both from OPD \& IPD) which was top in number in the list. Next 7 (29.16\%) came from Pus (both OPD \& IPD), followed by Blood 5(20.83\%) and CSF 2(8.33\%). (Table 4).

Two VRE came from TB \& Chest OPD urine samples. Twenty-two VRE came from IPD specimens. Among the wards Paediatric was on the top of the list. Six (27.27\%) VRE were from paediatric ward. In paediatric ward samples came from newborn up to 16 years age group children. Next highest no. of VRE positive samples i.e. 5(22.73\%) came from Medicine ward. Gynae \& Obs IPD sent 4(18.18\%) VRE. Three $(13.64 \%)$ VRE found in samples from Surgery ward. Three (13.64\%) VRE found in samples from Orthopaedics. One (4.55\%) VRE found in Pus sample of E.N.T. ward. (Table 5). Total no of HLGR Enterococci among 280 Enterococcal growth was 100 (35.71\%). Among 24 VRE 20 were also found to be HLGR. Among 100 HLGR Enterococci $6(6 \%)$ found in various clinical specimens from different OPD and 94((94\%) found in various clinical specimens from different IPD. Among HLGR Enterococci 60 (60\%) were E. faecalis and 40 (40\%) were E faecium.

In this study HLGR in Urine was at the top of the list (46\%) followed by Pus (25\%), Blood (23\%) \& CSF (6\%).

Fourty six HLGR Enterococci found in Urine among which 32 (69.57\%) were E. faecalis and 14 (30.43\%) were E. faecium. Twenty-five HLGR enterococci found in pus among which 15 (60\%) were E. faecalis and 10 (40\%) were E. faecium. Twenty-three HLGR enterococci found in blood among which 9 (39.13\%) were E. faecalis and 14 (60.87\%) were E. Faecium, Six HLGR enterococci found in CSF among which 4 (66.67\%) were E. faecalis and 2 (33.33\%) were E. faecium. In urine, pus \& CSF most of the HLGR were E. faecalis but in Blood most of the HLGR were E. faecium. (Table 6). Chi-Square test done and the difference of getting different species of HLGR in different samples found not significant. (d.f. $=3, p>0.05$ ). 


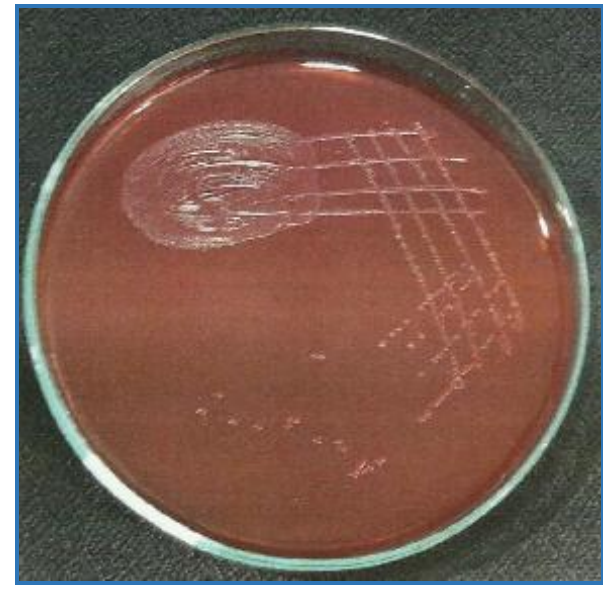

Figure 1. Enterococcal Colonies in MacConkey Agar Medium

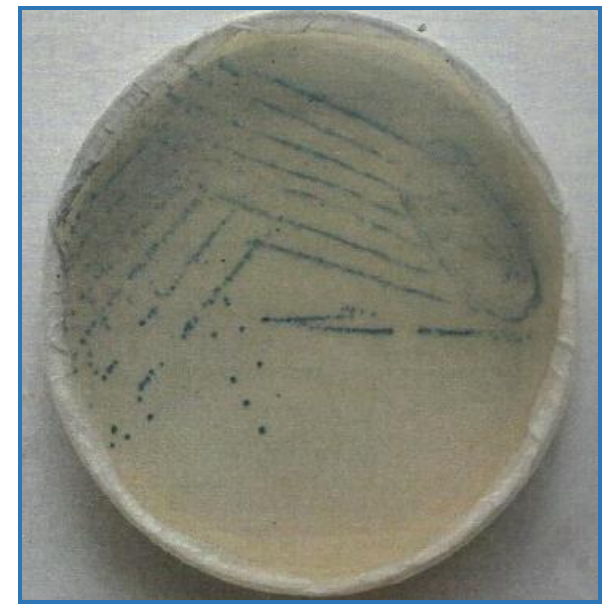

Figure 2. Enterococcal Colonies in Chromogenic Agar Medium

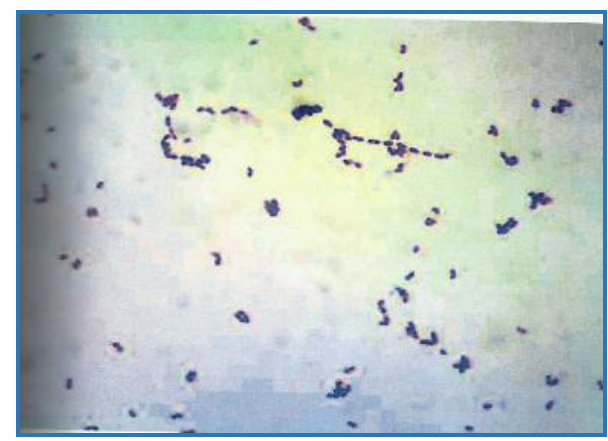

Figure 3. Morphology of Enterococci in Gram Strain

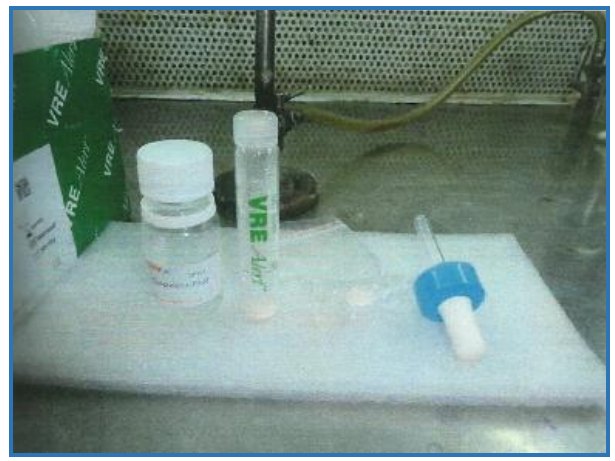

Figure 4a. VRE Alert Kit

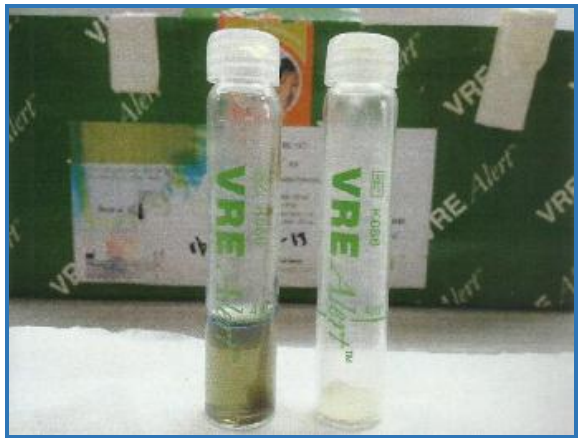

Figure 4b. VRE Alert Kit for VRE Screening Test

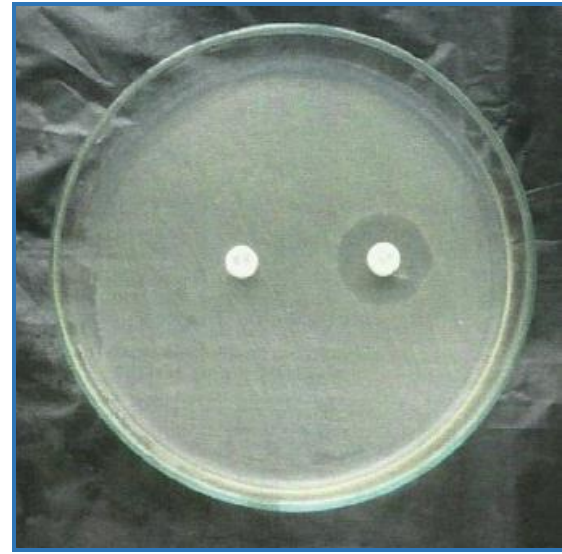

Figure 5. Vancomycin Resistant and High Level Gentamicin Sensitive Enterococci

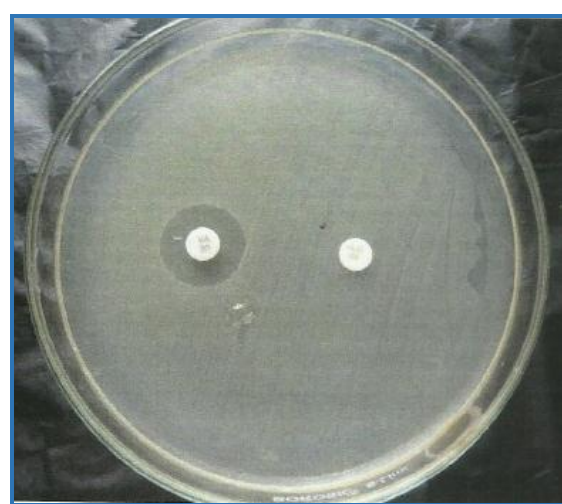

Figure 6. Vancomycin Sensitive and High Level Gentamicin Resistant Enterococci

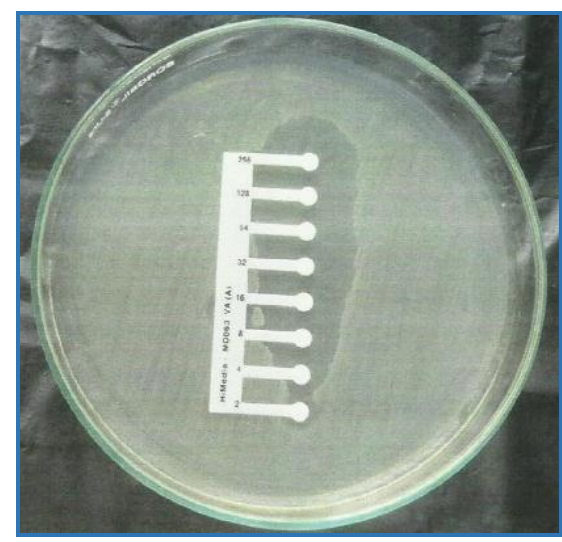

Figure 7. E-Test for Vancomycin of Enterococci. $\mathrm{MIC} \leq 2 \mu \mathrm{g} / \mathrm{ml}$ 


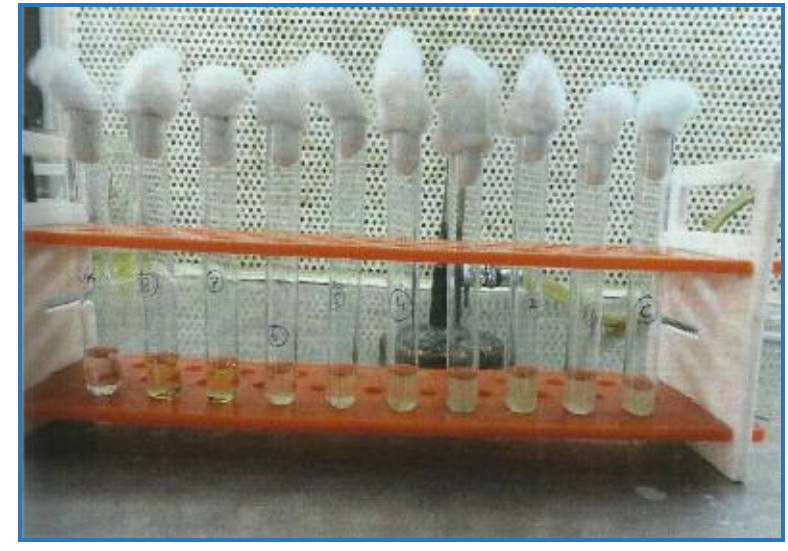

Figure 8. MIC done by Macro Dilution (Tube Dilution Method)

MIC $256 \mu \mathrm{g} / \mathrm{ml}$

\begin{tabular}{|c|c|c|}
\hline Organism & \multicolumn{2}{|c|}{ Positive Growth } \\
\hline & No. & \% \\
\hline E. Coli & 728 & 30.33 \\
\hline Staphylococci & 492 & 20.50 \\
\hline Klebsiella & 396 & 16.50 \\
\hline Enterococci & 280 & 11.67 \\
\hline Pseudomonas & 252 & 10.50 \\
\hline Proteus & 120 & 5.00 \\
\hline Acinetobacter & 48 & 2.00 \\
\hline Candida & 48 & 2.00 \\
\hline Citrobacter & 24 & 1.00 \\
\hline Corynebacterium Species & 12 & 0.50 \\
\hline Total & $\mathbf{2 4 0 0}$ & $\mathbf{1 0 0 . 0}$ \\
\hline
\end{tabular}

Table 1. Distribution of organisms in growth positive cultures in various clinical specimens under study

\begin{tabular}{|c|c|c|}
\hline \multirow{2}{*}{ Sample } & \multicolumn{2}{|c|}{ Frequency } \\
\cline { 2 - 3 } & No. & \% \\
\hline Urine & 1700 & 70.83 \\
\hline Pus & 350 & 14.58 \\
\hline Blood & 250 & 10.42 \\
\hline CSF & 100 & 4.17 \\
\hline Total & $\mathbf{2 4 0 0}$ & $\mathbf{1 0 0 . 0}$ \\
\hline \multicolumn{2}{|c|}{$\begin{array}{c}\text { Table 2. Distribution of specimens } \\
\text { showing growth under study }\end{array}$} \\
\hline
\end{tabular}

\begin{tabular}{|c|c|c|}
\hline \multirow{2}{*}{ Specimen } & \multicolumn{2}{|c|}{ Frequency } \\
\cline { 2 - 3 } & No. & \% \\
\hline E. Faecalis & 200 & 71.43 \\
\hline E. Faecium & 70 & 25.00 \\
\hline E. Duran & 4 & 1.43 \\
\hline E. Casseliflavus & 4 & 1.43 \\
\hline E. Gallinarum & 2 & 0.71 \\
\hline Total & $\mathbf{2 8 0}$ & $\mathbf{1 0 0 . 0 0}$ \\
\hline Table 3. Distribution of Enterococcal species \\
in various clinical specimens under study \\
\hline
\end{tabular}

\begin{tabular}{|c|c|c|}
\hline Specimen & \multicolumn{2}{|c|}{ Frequency } \\
\hline & No. & \% \\
\hline Urine & 10 & 41.67 \\
\hline Pus & 7 & 29.17 \\
\hline Blood & 5 & 20.83 \\
\hline CSF & 2 & 8.33 \\
\hline Total & $\mathbf{2 4}$ & $\mathbf{1 0 0 . 0 0}$ \\
\hline $\begin{array}{c}\text { Table 4. Specimen-wise distribution of } \\
\text { vancomycin resistant enterococci (VRE) }\end{array}$ \\
\hline
\end{tabular}

\begin{tabular}{|c|c|c|c|c|c|}
\hline Sample & $\begin{array}{c}\text { Urine } \\
\text { No. } \\
(\%)\end{array}$ & $\begin{array}{l}\text { Pus } \\
\text { No. } \\
\text { (\%) }\end{array}$ & $\begin{array}{l}\text { Blood } \\
\text { No. } \\
(\%)\end{array}$ & $\begin{array}{l}\text { CSF } \\
\text { No. } \\
\text { (\%) }\end{array}$ & $\begin{array}{l}\text { Total } \\
\text { No. } \\
\text { (\%) }\end{array}$ \\
\hline \multicolumn{6}{|l|}{ OPD $(n=2)$} \\
\hline Chest OPD & $2(100.00)$ & -- & -- & -- & $2(100.00)$ \\
\hline Total & $2(100.00)$ & -- & -- & -- & $2(100.00)$ \\
\hline \multicolumn{6}{|l|}{ IPD $(n=22)$} \\
\hline Medicine & $1(4.55)$ & & 3 (13.64) & $1(4.55)$ & 5 (22.73) \\
\hline Surgery & $1(4.55)$ & $1(4.55)$ & \begin{tabular}{|l|}
$1(4.55)$ \\
\end{tabular} & -- & $3(13.64)$ \\
\hline Orthopaedics & $1(4.55)$ & 2 (9.09) & -- & -- & $3(13.64)$ \\
\hline $\begin{array}{c}\text { Gynaecology } \\
\text { and } \\
\text { Obstetrics }\end{array}$ & $3(13.64)$ & $1(4.55)$ & -- & -- & $4(18.18)$ \\
\hline Paediatrics & $2(9.09)$ & $2(9.09)$ & $1(4.55)$ & $1(4.55)$ & $6(27.27)$ \\
\hline E. N. T. & -- & $1(4.55)$ & -- & -- & $1(4.55)$ \\
\hline Total & $8(36.38)$ & $7(31.83)$ & $5(22.74)$ & $2(9.10)$ & $22(100.00)$ \\
\hline $\begin{array}{r}\mathrm{Ta} \\
\text { teroco }\end{array}$ & n Differe & ent Clini & $\begin{array}{l}\text { Specime } \\
\text { r Study }\end{array}$ & 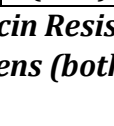 & 1 \\
\hline
\end{tabular}

\begin{tabular}{|c|c|c|c|}
\hline Specimen & E. faecalis & E. faecium & Total \\
\hline & No. (\%) & No. (\%) & No. (\%) \\
\hline Urine & $32(69.57)$ & $14(30.43)$ & $46(100.00)$ \\
\hline Pus & $15(60)$ & $10(40)$ & $25(100.00)$ \\
\hline Blood & $9(39.13)$ & $14(60.87)$ & $23(100.00)$ \\
\hline CSF & $4(66.67)$ & $2(33.33)$ & $6(100.00)$ \\
\hline Total & $60(60.00)$ & 40(40.00) & 100(100.00) \\
\hline \multicolumn{3}{|c|}{ Table 6. Species-wise distribution of high level } \\
gentamicin resistant enterococci (HLGR) \\
in various clinical specimens
\end{tabular}

\section{DISCUSSION}

Enterococci are currently ascendant nosocomial pathogen having the second most common organism from nosocomial UTI and wound infection and third most common cause of Bacteraemia. Again, Enterococci with high level aminoglycoside resistance, $\beta$-lactamase production and glycopeptides resistance including VRE have emerged posing a therapeutic challenge to physicians due to the ease of acquiring and transferring anti-microbial drug resistance. The present study was undertaken to determine the prevalence of vancomycin resistance as well as high level Gentamicin resistance in Enterococci isolated from urine, various pus samples, blood culture and specimens of cerebrospinal fluid in a tertiary care hospital at eastern part of India, Bihar. The specimens were collected from both indoor and outpatient department of this institution.

During this study period, total no of specimens received at laboratory 2788. All samples were put on culture. No growth of organism was found in 388 samples and growth of different organisms found in 2400 samples. Among the culture positive specimens, 280 Enterococci (11.67\%) were isolated. P.J. Desai et al (2001) found 22.19\% prevalence of Enterococci in various clinical specimens,(10) whereas Susil Kumar Jada et al (2012),(11) reported the prevalence of enterococci $15.46 \%$, among different organisms. Distribution of Enterococci positive specimens in indoors and outdoors of various departments: Indoor-266 (95\%), OPD-14 (5\%). Modi et al (2012) found $2.4 \%$ from OPD clinical specimens and 97.6\% from in patient department. Therefore, the findings of Modi et al(12) is comparable with the findings of present study. In the present study, the incidence of Enterococcal 
species were as follows: E. faecalis 200 (71.43\%); E. faecium 70 (25\%), E. duran 4 (1.43\%); E. Casseliflavus 4 (1.43\%); E. gallinarum $2(0.71 \%)$. Here, E. faecalis $(71.43 \%)$ was predominant enterococcal species, accounting for $71.43 \%$ of Enterococcal isolates and E. faecium had accounted 25\%. Similar results were observed in various studies. Ansari et al (2011)(13) from Pokhra, Nepal in their study found E. faecalis $52 \%$ and E. faecium 34\%, whereas Sengupta et al(14) from Kanchipuram, Tamil Nadu accounted $83.3 \%$ E. faecalis and $16 \%$ E. faecium. Uma Chaudhary et al(15) (2007) reported $72.3 \%$ E. faecalis; E. faecium $17.30 \%$; E. duran $2.3 \%$; E. casseliflavus $1.92 \%$ in various clinical specimens. P. J. Desai et al (2001) reported a prevalence of E. gallinarum 0.49\%; E. faecalis $49.50 \%$; E. faecium $35.64 \%$ and $E$. casseliflavus $0.49 \%$ in various clinical specimens. Historically, the ratio of infections due to $\mathrm{E}$. faecalis to those due to all other enterococci species was approximately 10:1. In recent years there has been a progressive decline in this ratio [Mundy L. M.; et al 2000](16) While E. faecalis remains the predominant species in clinical infection, E. faecium isolates are increasing in proportion. The trend is particularly true for blood isolates where the ratio of E. faecalis to E. faecium has been decreased from 3.7:1 in 1996 to 1.9:1 in 1999. [Edwards DD 2000].

Similar findings were observed in the present study i.e. 2.85:1. In present study out of 280 enterococcal isolates, 24 were vancomycin resistant Enterococci (VRE) i.e. 8.57\%. In our country, reports of the prevalence of VRE from different Institutions are as follows:

From Medical College, N. Delhi 8\%(17); from Chandigarh 5.5\%; from Mumbai 23\% (Sood et al 2007)(18); from Ahmedabad (Modi et al 2012) 4\%; Sengupta et al from SRM Medical College, Kanchipuram 16.7\%(13); Bose et al in the year 2011 from NRS Medical college, Kolkata; VRE 3.5\%(19); Mukherjee $\mathrm{K}$ et from the same medical college found 3.8\% VRE in clinical specimens all of which were sensitive to Linezolid in the year 2016.(20) Incidence of VRE infection in European countries is relatively low (1-3\%) compared with high and rising rate in U.S. The rates have steadily increased from 0.3 to 7.9 [CDC-1993].

In this study, High Level Gentamicin Resistance (HLGR) in enterococci was seen $100(35.71 \%)$ isolates. And this was more in E. faecalis $60(60 \%)$ than E. faecium $40 \%$ (40). Out of 24 VRE, 20 (83.33\%) were HLGR Enterococci.

Suman Poddar et al(21) from R. G. Kar Medical College, Kolkata (2011) found $40.04 \%$ enterococci isolates as HLGR. Among the resistance strains the predominant strains was E. faecalis $40 \%$, followed by E. faecium $25 \%$.

Chandra Sengupta et al SRM Medical College, Kanchipuram, Tamil Nadu found 50\% Enterococci resistant to Gentamicin.

Modi et al (2012): reported 23.6\% HLGR enterococci.

Anjana Telkar et al (2012) reported 68\% HLGR enterococci isolated from blood stream.

VRE is now being seen with increasing frequency among patients with chronic renal failure, cancer, organ transplant recipients and patients who experience prolonged hospitalization. They tend to occur in more debilitated or seriously ill hospitalized patients.

Vancomycin most probably predisposes patients to colonization and infection with VRE by inhibiting the growth of the normal Gram-positive bowel flora and by providing selective advantage for VRE that may be present in small numbers in the individual's bowel.

The emergence of VRE has alarmed the global infectious disease community for several reasons. Because of the limited therapeutic option for treating serious infections caused by enterococci, it has emerged as one of the leading clinical challenges for physicians. The limited successes over the past decade of prevention and control strategies for controlling vancomycin resistance (As well as methicillin resistance in staphylococcus sp.) highlight the difficulty of limiting the problem once it is established.

\section{CONCLUSION}

Therefore, in the present study, out of all culture positive clinical specimens, the prevalence of enterococci was $11.67 \%$ and among these enterococci, VRE were $8.57 \%$. This figure occupies a position in between European studies (1-3\%) and USA (0.4-13.6\%) studies. The percentage of VRE in the present study $(8.57 \%)$ shows that the incidence remains in static position in India (Indian Scenario-3.5-23\%).

In this study HLGR enterococci was $35.71 \%$, which also occupies the position among other Indian studies. Modi et al (2012) reported 23.6\% HLGR and Lata Kapoor et al (2005)(22) reported 66\% HLGR enterococci. Although eradication of VRE may not be possible in institutions where VRE are endemic, efforts to reduce the prevalence of colonization and infection should continue.

Guidelines have been established by CDC to prevent the spread of Vancomycin Resistance Enterococci. The principle recommendations are-

1. The prudent use of Vancomycin.

2. An ongoing education program for all hospital staff about the threat of VRE.

3. A co-operative effort between health care providers and hospital microbiology laboratory personnel that will allow VRE to be promptly and accurately detected.

4. The implementation of appropriate infection control measures to prevent person to person spread of VRE.

5. Screening of health care workers in order to identify carrier rates.

6. Surveillance culture in high prevalence area such as intensive care units and operation theatres are immediate requirements in order to keep the spread of VRE under control.

The steady pandemic spread of VRE along with acquisition of resistance to newer antimicrobials warrants continued surveillance of these versatile pathogens. Innovative intervention strategies and multifaceted programs that make into consideration the local epidemiology of the spread of VRE may yield the best outcomes.

\section{REFERENCES}

[1] Mda CG, Steigenualt AG, Morey RE, et al. Characterizatiiru if three new enterococcal species, enterococcus sp. nov. CDC PNS-E1, enterococcus sp. nov. CDC PNS-E2, and enterococcus sp. nov. CDC PNSE3, isolated from human clinical specimens. J Clin Microbiol 2004;42(3):11992-8.

[2] Teixeria LM, Facklam RR. Enterococcus. In: Borriello SP, Murray PR, Funke G, (eds). Topley \& Wilson's microbiology \& microbial infections. Bacteriology $10^{\text {th }}$ edn. Hodder Arnold, London 2005:882-902. 
[3] Mittal S, Singla P, Bala K, et al. Vancomycin and high level aminoglycoside resistance in enterococcus species in a tertiary health care centre: a therapeutic concern. J Pathogens 2016:5.

[4] NNIS System. National nosocomial infections surveillance (NNIS) system report, data summary from january 1990-may 1999, issued june 1999. A report from the NNIS system. Am J Infect Control 1999;27(6):520-32.

[5] Mathur P, Kapil A, Chandra R, et al. Antimicrobial resistance in enterococcus faecalis at a tertiary care centre of northern India. Indian $\mathrm{J}$ Med Res 2003;118:25-8.

[6] Telkar A, Mahesh BC, Raghabendra VP, et al. Change in the prevalence and the antibiotic resistance of the enterococcal species isolated from blood cultures. Journal of Clinical and Diagnostic Research 2012;6(3):405-7.

[7] Chow JW. Aminoglycoside resistance in enterococci. Clin Infect Dis 2000;31:586-9.

[8] Patricia M. Bailey \& Scott's diagnostic microbiology. 13th edn. P:247-264. http://evolve. elsevier.com.

[9] Methods for dilution antimicrobial susceptibility tests for bacteria that grow aerobically. National committee for clinical laboratory standards. $5^{\text {th }}$ edn. Approved standard M7-A5. Wayne (PA) 2000.

[10] Desai PJ, Pandit D, Mathur M, et al. Prevalence, identification and distribution of various species of enterococci isolated from clinical specimens with special reference to urinary tract infection in catheterized patients. Indian Journal of Medical Microbiology 2001;19(3):132-7.

[11] Jada SK, Jayakumar K. Prevalence of enterococcus species from various clinical specimens in shri sathya sai medical college and research institute with special reference to specification \& their resistance to vancomycin. International Journal of Medical and Clinical Research 2012;3(4):154-60.

[12] Modi GB, Soni ST, Patel KJ, et al. Prevalence of vancomycin resistant enterococci in tertiary care hospital, Western, India. International Journal of Medical and Clinical Research 2012;4(2):182-5.
[13] Ansari MT, Raj BD, Abhisshek G, et al. Department of microbiology. Manipal college of medical sciences, Pokhara, Nepal. Bo-2. Microcon 2011.

[14] Sengupta C, Sangamithra V, Madhavan R, et al. Identification, speciemen and antibiotic susceptibility testing of enterococcus from various clinical specimens. A tertiary care hospital BP-75 MicroCon 2011.

[15] Chaudhary U, Shamma M, Yadav A. Antimicrobial susceptibility patterns of common and unusual enterococcus species isolated from clinical specimens. J Infect Dis Antimicrob agents 2007;24:55-62.

[16] Mundy LM, Sahm DF, Gilmore M. Relationships between enterococcal virulence and antimicrobial resistance. Clin Microbiol Rev 2000;13(4):513-22.

[17] Sood S, Kapil A, Das B, et al. A five-year study of positive blood cultures: with emphasis on polymicrobial bacteraemia. Indian Practitioner 1997;50(7):575.

[18] Sood S, Malhotra M, Das BK, et al. Enterococcal infections \& antimicrobial resistance. Indian J Med Res 2008;128(2):111-21.

[19] Bose M, Chatterjee SS, Chakraborty B, et al. A study of enterococcal urinary tract infection in a tertiary care hospital in Eastern India. MSP-16. MicroCon 2011.

[20] Mukherjee K, Bhattacharya D, Chakrabarti G, et al. Prevalence and antibiotic susceptibility pattern of enterococcus species from various clinical samples in a tertiary care hospital in Kolkata. Int J of Contemporary Medical Research 2016;3(6):24547379.

[21] Poddar S, Ghose RR, Kumar S, et al. Status of high level aminoglycoside resistance in enterococcus species in a tertiary care hospital in Eastern India. AMP-35d. BHU Varanasi, MicroCon 2011.

[22] Kapoor L, Randhawa VS, Deb M. Antimicrobial resistance of enterococcal blood isolates at a pediatric care hospital in India. Jpn J Infect Dis 2005;58(2):1013. 